\title{
Pteknokultura
}

\#ERREJÓn Galván, I. (2015). La construcción de un sujeto popular. Revista Teknokultura, Vol. 12(1), 39-53.

Recibido: 21-02-2015

Aceptado: 09-03-2015
Open peer review:

http://revistas.ucm.es/index.php/TEKN/pages/view/opr-260

\section{La construcción de un sujeto popular}

\author{
Constructing a popular subject \\ Íñigo Errejón Galván ${ }^{1}$ \\ Podemos \\ ierrejon@cps.ucm.es
}

\section{Resumen}

En esta entrevista Iñigo Errejón relata su intensa trayectoria política que nace en una familia militante antifranquista, que conoce la década de gobiernos progresistas latinoamericanos y piensa desde la distancia la política española. Dialogamos con el joven politólogo que hace un año planteó la hipótesis Podemos y hoy es Secretario Político del nuevo partido que promete acabar con el bipartidismo en el 2015.

\section{Palabras Clave}

Podemos, ideologías, identidades, teoría crítica, América Latina, crisis, populismo.

\footnotetext{
${ }^{1}$ Responsable de la Secretaria Política del Consejo de Coordinación de Podemos.
} 


\section{Abstract}

In this interview, Iñigo Errejón describes his intense political career, which starts in an anti- Franco family of political activists, followed by his experience of the decade of progressive governments in Latin America whilst observing Spanish politics from afar. This is a conversation with the young political scientist who set out the Podemos Hypothesis and has become the Political Secretary of the new party that promises to put an end to the two-party system in Spain in 2015.

\section{KeYWORDS}

Podemos, ideologies, identities, critical theory, Latin America, crisis, populism. 
Ariel Jerez (AJ): Hola Íñigo, queremos hacerte una primera pregunta para tener perspectiva biográfica de la formación de la conciencia política de nuestros entrevistados, ¿cuáles consideras que son tus elementos de socialización política, el primer momento "político" que recuerdes?

Ínigo Errejón (IE): En mi caso viene de mis dos padres, los dos militantes contra la dictadura, los dos integrantes de organizaciones de la izquierda radical de finales de los años 60 y principios de los 70. Uno de mis primeros recuerdos políticos es mi madre durmiéndome y cantándome la "Grándola, Vila Morena" cuando era muy pequeñito, canciones de la Guerra Civil, del bando derrotado claro.

Son cosas que yo tenía naturalizadas y que luego, en un momento dado, uno las empieza como a asimilar como toma de posición: mis primeras fotos en la calle son en movilizaciones, en las del referéndum contra la entrada de España en la OTAN [Organización del Tratado del Atlántico Norte], que yo creo que tenía tres años. Los 1 de Mayo, ir a las movilizaciones como un plan familiar, como cuando uno se va al cine o cuando antes se iba a la casa de campo a comer tortilla. $\mathrm{O}$ sea que son un habitus familiar, o casi.

\begin{abstract}
AJ: Claro.
IE: También la conciencia de mi padre, represaliado durante la dictadura. Luego enseguida, viene la politización cuando soy adolescente, que empieza en otros ámbitos en la generación de los 90, con la música, con los fanzines, con la contracultura. En mi caso, en primer lugar con una militancia en el ámbito libertario, y en paralelo, o solapándose, en el movimiento estudiantil. Cuando entro en la Facultad de Políticas y Sociología de la Complutense, si uno viene de un bagaje familiar politizado y con compromiso militante ya antes de entrar, esta Facultad es como el esplendor. Y el estallido: a partir de ahí vienen unos años muy acelerados de carrera, febriles, en los que por una parte le echo muchísimas horas a la militancia pero a la vez lo estudias en clase. Entonces empiezas a iniciar un diálogo, al principio con desencuentros y luego en espacios intermedios de discusión entre lo que aprendes en el aula o leyendo, y lo que practicas, lo que tocas, lo que discutes con los compañeros. Después viene el periodo de movilizaciones antiglobalización, sobre todo de la segunda legislatura del gobierno del Partido Popular, el movimiento del No A La LOU [Ley Orgánica de Universidades], el Prestige, la
\end{abstract}


Huelga General... Y el No A La Guerra como momento cumbre de movilización de masas con la experiencia de desobediencia pacífica que nos interesa y nos toca mucho.

Luego constituyen un salto en conocimiento académico e intelectual, pero también de observación participante y militante los procesos políticos de signo popular en América Latina. Para mí supone un cambio drástico en la forma de pensar el compromiso, de pensar la política, pero no sólo de pensarla como militante, también de pensarla como...

\section{AJ: ¿También en términos teóricos?}

IE: En términos teóricos, como analista, como investigador. Me paso muchos años a caballo entre América Latina y Europa, incluso si estoy en Europa hago la tesis sobre el proceso político latinoamericano, en este caso el boliviano. Leo mucho y juego durante mucho tiempo a probar a ver si algunas de las categorías, de las palabras o de los elementos de análisis que sirven allá, sirven también para pensar algo de lo que nos pasa aquí. No por pensar que las situaciones son iguales, pero sí por desafiar una cierta visión colonial según la cual las categorías se fraguan en el Norte, y a veces en el Sur se aplican de forma malformada del "fenómeno" puro que está en los sistemas políticos del Norte.

\section{AJ: En el Sur interpretada como "anomalía".}

IE: En el Sur hay "anomalías" mezcladas, mestizas, siempre impuras, siempre un poco desastre. Entonces juego a decir "aquí está habiendo procesos de cambio político, procesos constituyentes, procesos de reforma del Estado muy ricos y muy profundos, con muchas tensiones y con muchas contradicciones. Son materias primas y sociedades muy diferentes, pero que a lo mejor también valen de regreso a acá; es una operación intelectual que no se entiende muy bien, o que no se comparte. Cuando empezamos a hablar aquí de "crisis de régimen", de "proceso constituyente", cuando empezamos a reivindicar un entendimiento de la posibilidad del retorno de la palabra "pueblo" como referente colectivo de una mayoría que se siente maltratada pero no representada, toda esa fase, creo que se vive aquí como con una cierta distancia, o incluso malestar estético diciendo: "Nosotros ya hemos superado eso, somos una sociedad europea". Y eso dura prácticamente hasta que ya estamos puestos en marcha, trabajando en la "hipótesis Podemos", que demuestra su enorme capacidad de acumulación política y de abrir posibilidades que parecían cerradas, y ahí ya la cosa cambia. 


\section{AJ: En el Seminario de Cartografías de Culturas Radicales manejamos la hipótesis de que, como consecuencia del franquismo la cultura militante ha quedado reducida en un muy pequeño espacio híper politizado, en una sociedad altamente despolitizada que tiene muchas dificultades para poner en marcha dinámicas de participación de una sociedad civil un poco más autónoma. Yo creo que en buena parte de la discusión que se está dando en Podemos se acumulan problemas pendientes no debatidos por la izquierda desde hace muchas décadas.}

IE: Las experiencias del fascismo en Europa han echado a muchos sectores, también sectores de la izquierda, en manos de un mantra liberal según el cual los sujetos colectivos y universales, las pasiones colectivas y de masas, conducen necesariamente al totalitarismo y a la cancelación del pluralismo. Y no hay forma de conciliar compromisos colectivos, sujetos colectivos trascendentes y pluralismo, derecho a la diferencia y a la disidencia, y por eso algunos se asustan. Estamos viviendo ataques también de intelectuales del centro-izquierda en España, o que fungían por ser intelectuales del centro-izquierda, de una agresividad que sólo puede nacer de una profunda incomodidad con respecto a la hipótesis Podemos.

Por otra parte, hay algo que tiene que ver con elementos generacionales, con elementos de incomprensión y con desacuerdos. Pero hay otra que tiene que ver con la imposibilidad de imaginar que puede haber un retorno del sujeto "pueblo" a la política que no tenga consecuencias totalitarias.

Por otra parte, creo que en España, el estallido que a nivel de todo el mundo es "el 68”, aquí resuena pero no es el 68 en Francia y en México, el 69 en el Cordobazo en Argentina, el 69 en Italia, y en "el 77" italiano. Es un ciclo de protesta y de reinvención radical de la política en términos muy positivos, según los cuales lo político es bueno y hay que hacer política y la gente tiene que hacer política y hay que reivindicarla. Pero después de esa explosión, viene una oleada de repliegue conservador, donde decir que algo está politizado, es decir algo malo. Un contexto donde el individuo verdaderamente inteligente, es un individuo que se repliega a lo privado, que le regala lo político y lo público a las pequeñas minorías no elegidas por nadie, y que es un individuo siempre desconfiado de lo colectivo, siempre miedoso, y que es héroe en lo individual, idiota en lo colectivo. Y eso, yo creo que en España tiene que ver con un cambio de régimen bien hecho porque es capaz de producir esta habilidad, repartir las posiciones y construir un orden que permanece muy poco alterado durante treinta años. Con una transición que en términos de Gramsci, sería una buena experiencia de revolución pasiva, las élites del régimen anterior, la dictadura, para seguir gobernando tienen que incorporar una parte de las élites políticas, de los 
intelectuales y de las demandas de los grupos subalternos. Régimen con importantes derechos civiles y democráticos, con importantes conquistas sociales para las capas populares, pero integradas dentro de un edificio en las que la oligarquía del régimen anterior no pierde los mandos fundamentales. Y el relato que acompaña todo eso, creo que es un relato que además nosotros lo vivimos como fuertemente generacional: "La historia ya está hecha, váyanse a casa. Además con un relato particularmente mentiroso, porque de repente es un relato en el que todo el mundo queda exculpado. Aquí no hubo compromiso, no hubo militantes, no hubo torturas, no hubo presos, no hubo gente que colaboró con la dictadura, aquí lo que hubo fue un régimen un poco viejo y una juventud "ye-ye", en la que todo el mundo corría delante de los grises. Y eso construye un consenso muy sólido, un reparto de posiciones que permanece casi inalterado y construye una cierta despolitización, porque vacía la política de las pasiones, que se refugia en otros lugares: en el fútbol, en el ocio. El problema es que esa construcción hoy no da más de sí para explicar las carencias o las expectativas frustradas de importantes sectores de españoles. Resulta que tenemos cientos de miles de jóvenes que se tienen que ir del país porque no encuentran empleo, cinco millones y medio de parados, los derechos sociales son papel mojado en una constitución, que sin embargo no se podía tocar, pero los poderes fácticos han modificado a través del PSOE y del PP sin que los ciudadanos puedan votarla. Resulta que cuando están siendo las marchas por la dignidad en Madrid el 22 de marzo, que están reivindicando cosas enormemente universales, que todo el mundo comparte como de sentido común, mientras pasa esto, las élites del régimen del 78, están velando a Adolfo Suárez. Y el discurso es: "Necesitamos aquél espíritu de consenso". Y eso no funciona, no porque a la gente le parezca mal lo que hiciera Adolfo Suárez, sino porque Adolfo Suárez, como objeto político, ya no explica nada de la España de hoy. Así que la gente dice: "Muy bien, perfecto, hicieron ustedes la mejor transición del mundo, todo fue fantástico y hubo grandes hombres" (no la acción popular, nunca: un relato sólo de élites). Sí, hubo grandes hombres que articularon importantísimos consensos que nos han permitido... Muy bien, si es que no se los discuto, pero, ¿qué tiene eso que ver con mis necesidades insatisfechas hoy? Ahí las élites se han encontrado frente a una marcada crisis de imaginación. La vivían con una resignación tranquila hasta el 25 de mayo.

Llega el 25 de mayo y de repente, Podemos, con un resultado muy modesto, porque el resultado de Podemos es muy modesto para la situación en la que está España, desata una carrera entre las élites para parecer nuevas, para vestirse con ropas nuevas. Rajoy y sus planes de regeneración democrática y de lucha contra la corrupción, el Partido Socialista eligiendo un 
líder nuevo al que le tienen que remangar las mangas de la camisa, y le tienen que quitar la corbata, y le tienen que hacer hablar como otra cosa, para hacerle parecer una cosa nueva. En Izquierda Unida suceden cosas similares aunque con un poco más de retraso. No hay fuerza política que no acuse el movimiento que se le pega al tablero la noche del 25 de mayo de 2014. Que es un movimiento más importante en términos cualitativos que cuantitativos. En términos cuantitativos, 1.250 .000 votos, para una candidatura con ese mimbre tan débil en la organización y en lo económico, es mucho. Pero es poco para la que está cayendo en el país. Lo fundamental es cómo han cambiado los términos de la discusión política en España; las élites ya no tienen el monopolio de ponerle los nombres a las cosas. Ya no fabrican ellos, en solitario, las explicaciones. Y eso creo que habla de un cierto retroceso cultural y también de un regreso de la pasión y la ilusión política. De una suerte de venganza plebeya, democrática, no violenta, pero decir: "Unos pocos durante mucho tiempo han acumulado mucho poder. La gente como yo, tradicionalmente sin poder político, que solo puede resignarse o votar entre las opciones preestablecidas, podemos hacer cosas".

\section{AJ: Hay una sensación de superación de cierto miedo de nuestra cultura política. Aunque en ciertas culturas profesionales e institucionales, la obediencia y la impunidad, el acomodarme a lo que me mandan, sin querer saber qué responsabilidad tengo en su resultado democrático, mantiene una base importante de despolitización, ¿no?}

IE: Creo que los fenómenos de despolitización funcionan, en primer lugar, como una dinámica de la privatización de los conflictos. Es decir, si te pasa algo es tu culpa, o te pasa exclusivamente a ti. Ahí tenemos la ruptura de aquellos conceptos, mitos, canciones, elementos estéticos y simbólicos de pertenencia, que permitían poner en contacto diferentes dolores. Y hay ahí toda una maniobra de retroceso cultural, de destrucción del sentido de pertenencia. Cuando Podemos irrumpe, se encuentra a mucha gente mayor, que llevaba sin militar, o con militancias medio subterráneas, veinte, treinta años, pero hay mucha gente sin experiencia política, casi hay que empezar de cero. Le presto mucha atención a estos temas; en los actos públicos que ha hecho Podemos se ha ido probando con diferentes canciones y nos hemos encontrado con la dificultad de que no hay canciones de nuestra generación. Ha tenido que ir o a canciones viejas, anteriores, de las generaciones anteriores, que a algunos por nuestra biografía nos emocionan pero no interpelan con la suficiente fuerza y con la suficiente amplitud al conjunto de la gente que está mirando a Podemos, o canciones de otros países. O sea, "Grándola vila morena", "L'Estaca", que son canciones que es que no son ni en castellano. Y eso, creo, tiene que ver con 
una construcción de un terreno cultural mínimo, de unos mimbres a partir de los cuales uno pueda reconstruir sujetos colectivos.

Eso por un parte, por otra parte creo que ha habido lo que se anunciaba en la postpolítica: una captura de la política por la gestión y la técnica. Una institucionalización extrema, que ha privado de "sex appeal" y de atractivo a la política. Creo que eso se empieza a quebrar con el cambio cultural que empieza con 15M; se abre una posibilidad de contestación de esa percepción.

\section{AJ: Junto a esto hay un reparto, generacionalmente diferenciado, de nuevos procesos de aprendizaje y de participación. Pareciera que Podemos debe atender, resolver y ecuacionar amplias expectativas de participación.}

IE: Hay que recordar que buena parte de los sectores activistas, hasta el 25 de mayo, tenía una sensación de que estamos en un impase. Creo que teníamos dos interpretaciones igualmente erróneas. Una muy mecanicista de lo social, digamos marxista o neo marxista, según la cual todavía no hay traducción política pero que lo político siempre va después de lo social. En una separación, que siempre la he entendido como parte de la separación liberal, donde hay un social al margen de lo político, donde vamos "ganando". El movimiento de las plazas va ganando porque estamos cambiando muchas cosas en un sentido molecular de lo micro, que ya producirá un cambio político. El cambio político, en última instancia, es lo de menos porque como solo es un resultante, lo importante es lo que pasa molecularmente. En el otro extremo, está el diagnóstico de que aquí todo está fatal, que no se consigue nada.

Sin embargo, lo que el 25 de mayo plantea no es que las cosas estén bien, pero claramente hay más grieta, hay más espacio, hay más recorrido del que parecía. Y en cuanto empieza eso, entramos en un tiempo político acelerado, todo el mundo llega a este momento político con una mochila muy cargada de expectativas, pero también de bajas pasiones, frustraciones y tristezas acumuladas. Creo que tiene una parte buena y una parte mala, la parte buena sería la impaciencia revolucionaria: "es lo que quiero, y lo quiero ya, este es el momento de la gente corriente como yo, y quiero que las cosas sean ya”. Pero tiene también la parte mala, un cierto comportamiento de consumismo individualista: “ ¡Mire esto es lo que yo quiero, satisfágamelo absolutamente ya!". Además desde que tenemos redes sociales esto se ha multiplicado: en su Facebook todo el mundo tiene siempre razón, todo el mundo sabe cómo se deberían hacer las cosas. Siempre digo lo mismo a los compañeros: Podemos no es la solución a todos los problemas. Podemos va a librar una serie de disputas y lo va a librar con la inteligencia, la 
fuerza y la generosidad de quienes la pongan a su servicio. Pero no va a solucionar todo el resto de problemas. En política creo que lo que diferencia a un opinador de un militante es coger las hipótesis, fabricarles piernas y ponerlas a andar. Esto no es, en modo alguno, un intento de quitarle peso a la teoría: la teoría es fundamental.

AJ: Sí, sin lugar a duda. Nos situamos entonces en los interiores de la hipótesis Podemos, en el sustrato teórico del populismo. ¿Qué es la crisis populista? ¿Cómo la pensamos para España, con qué elementos analizamos la subordinación política, de una relación con actores extranjeros, que es difícil que pueda entenderse como patriota, pero no llega a ser como en países coloniales? ¿Aquí también hay una dislocación o fragmentación en la capacidad de las élites, de la oligarquía en particular, de reivindicar para ellos la cuestión “nacional” de España? ¿Cuál es la traducción de un relato nacional-popular, en un país plurinacional y más ciudadano que popular?

IE: Aquí el avance de las instituciones y la construcción del Estado es sólido, hay ciudadanos, se confía en las instituciones, hay orden público, seguridad en la calle, el Estado produce orden y certeza: eres un ciudadano que para el problema uno va a la ventanilla uno, que para el problema dos va a la ventanilla dos y para el problema tres va a la ventanilla tres. Hice la tesis sobre Bolivia. En Bolivia la forma principal de reivindicar algo no es ir a ningún Ministerio o a ninguna institución pública. Es cortar la carretera antes de haber probado ninguna otra cosa. Aquí un grupo de ciudadanos solo se animan a cortar un carretera si han probado todo y no funciona, si entre ellos hay alguno que tenga algo de memoria de activismo y si logran vencer las desconfianzas. No digo que sea necesariamente mejor, estoy haciendo análisis: no postulo qué es mejor. Claramente eso construye un ethos ciudadano, más que un ethos popular, que tiende a ser más individualista, más desconfiado, menos dado a la entrega a lo colectivo, a esa pasión altruista que a veces puede estar en los inicios de la política.

De todas formas la crisis cambia todo, entre todo un poco en quiebra. Porque ha habido muchos ciudadanos que han visto que, por mucho, que vayan a la ventanilla uno, dos y tres a tu vecina la echan de la casa, que la van a desahuciar para darle la casa a un banco. La institucionalidad ya no responde tan bien como antes. Y ese dar respuesta es tanto satisfacerlas administrativamente, como generar un relato que las integre, que les prometa su satisfacción en el medio o largo plazo. Ese descontento emerge de forma correlativamente inorgánica, fragmentaria, desordenada, tumultuosa. 
Ese descontento está como huérfano: cuando eso sucede hay posibilidades de que haya nuevos actores, nuevos liderazgos, nuevos discursos, incluso nuevos palabras que sean capaces de producir una identificación popular nueva. Al principio es una identificación que lo primero que tiene en común no es tanto ningún interés objetivo, de clase, de nación, de religión, sino una sensación de maltrato colectivo, de no representación por parte de la elites y del poder. Por tanto, siempre hay un ánimo destituyente inicial, que luego va cristalizando en la construcción de un sujeto popular que puede hacer esa maniobra hegemónica por la cual una parte reclama ser el todo, diciendo: "Nosotros somos el pueblo y no estamos representados".

Se entreteje en esa identificación con referentes que siempre tienen una parte nueva y una parte heredada. Líderes nuevos que cuando existe mucho desprestigio de la política, su virtud principal es que vienen de fuera de la política, o que son percibidos como que viene de fuera de la política. Con referencias nuevas, nuevas palabras, ordenaciones nuevas. Atraviesan el tablero y lo reordenan. En nuestro caso, decimos que izquierda y derecha ya no es más la frontera que define lo importante para los ciudadanos, las posiciones que los ciudadanos van a tomar; es más importante ciudadanía-casta, por ejemplo.

Se han alterado las viejas formas políticas, pero lo que para algunos es un sinónimo de despolitización, para otros lo es de subversión. El populismo siempre tiene esta cosa curiosa que para los privilegiados puede ser subversión, pero una parte de la izquierda dice "esto no estaba en el manual, esto es nadar en sentido contrario". A veces, sin tener la menor complicidad de intereses, pueden incluso colocarse en el escenario como obreros privilegiados.

Para el caso de España, claro, reviste muchas complejidades que a mí me resultan apasionantes. ¿Por qué? Ese relato no puede anclarse en una identidad nacional española fuerte porque su hegemonización tradicional en las últimas décadas ha sido de la derecha. Y esto no ha sido solo una operación discursiva, porque hay mimbres históricos muy largos por los cuales la nación española tiene un vínculo concreto con la bandera nacional. Formalmente esa bandera nos representa a todos, pero no la vemos en las manifestaciones contra la guerra o en las huelgas generales por defender unas condiciones de trabajo más dignas, pero sí está, y muy presente, en las manifestaciones contra el derecho de las mujeres a decidir sobre sus embarazos o contra el derecho de los homosexuales a casarse, por ejemplo. Lo que signa claramente que aquí hay un conflicto nacional, en el sentido de resolver qué cosa queremos que sea la Nación.

Ese es uno de los problemas, el otro es la cuestión de la plurinacionalidad. Ha habido una construcción de la nación española como una identificación a la contra, de catalanes, de vascos, 
en mucha menor medida de gallegos, sobre una idea muy centralista y esencialista, según la cual no puede haber Nación española si convive con otras nacionalidades, naciones, o formas de sentirse nación, de querer ser un país. Resolver esas dos cuestiones a la carrera es muy complicado, de hecho está todavía por resolverse. Lo que pasa es que la crisis ha introducido elementos nuevos en esta cuestión. Por una parte ha introducido una disimulada subordinación de los poderes españoles a poderes extranjeros no elegidos, que pone de manifiesto un rol, en lo político y económico, semicolonial. Así que, por mucho que el gobierno del Partido Popular haga más grande la bandera de la Plaza Colón, es claro que cuando viene la señora Merkel aquí viene como la gobernadora de una provincia. Viene a su colonia y le rinden pleitesía. Lo que les hace mucho más difícil a las élites, a una parte de la oligarquía española, encarnar el interés nacional, de decir "el interés del país soy yo".

Hay una dislocación de la capacidad de las élites de reivindicar para ellos la cuestión de España. Pero es muy difícil pensar cómo, cuál es la traducción de un relato "nacional-popular" en un país plurinacional, y más ciudadano que popular. El avance de las instituciones y la construcción de un Estado sólido han producido un tipo de sociedad en la que los ciudadanos son más ciudadanos individuales que sujetos colectivos. La gente está más acostumbrada a solucionar sus problemas individualmente, como consumidor o como cliente-usuario. El Estado produce orden y certeza, aunque es un orden y certeza que dificulta y desincentiva la necesidad de asociarse porque las cosas hasta ahora funcionaban así.

En la crisis la institucionalidad ya no responde tan bien como antes. Lo que nos obliga a pensar cuándo se produce el momento de ruptura populista, cómo se construye un nuevo sujeto y un nuevo proyecto siempre intermediado por un Estado que en España funciona y es sólido.

\section{AJ: Hacer emerger lo popular parece una tarea compleja, porque en España durante el franquismo lo popular se construye desde una particular folklorización que lo ancla a determinados componentes.}

IE: Y desdeñada por cosmopolitas, que son un poco de izquierdas. Conecta con una idea trágica de lo español, según lo cual ser verdaderamente progresista es irte a París. Pero no bailar un chotis. No digo que haya que recuperar el chotis.

\section{AJ: De un popular goyesco.}


IE: De un popular goyesco, y como chabacano, no sé. Que le regala lo popular a los sectores más reaccionarios. Tiene que ver con esa suerte de cosmopolitismo y de aristocratización de los sectores progresistas, que están mirando más qué ocurre en Londres, qué ocurre en Berlín, qué ocurre en no sé dónde, pero que desatienden la construcción de elementos culturales de lo popular, el patrimonio colectivo a partir de los cuales tejer una narrativa de pueblo.

\section{AJ: El País estilo ha hecho lo suyo [ríe]. \\ IE: Claro, claro, claro.}

A. J.: Retomando esa crisis populista, tejida sobre esas demandas insatisfechas, anonimizadas por la no representación elitista, a la búsqueda de un líder que sea capaz de tejer la trama que los incluya. Por la propia tradición ideológica de las izquierdas españolas, el liderazgo se presenta como un tema controvertido. Es curioso no tener presente que en política el vacío no existe, que siempre hay un liderazgo, que podrá ser peor o mejor, pero siempre lo hay, ¿no?

IE: Podrá ser explícito o implícito.

AJ: Pero siempre lo hay. ¿Cómo se puede trabajar esa idea del liderazgo, cómo puede ser explicado el papel muy diferenciado que juega para los distintos públicos a los que va dirigido? De hecho, mucha gente puede tener una vinculación prioritariamente emocional al liderazgo, otros más reflexiva, otros de desconfianza fiscalizadora; pero no quiere decir que la potencia política del liderazgo no pueda operar para todos ellos en un mismo espacio.

IE: Es otra de las cuestiones complicadas. Hay una premisa antes que nada: el esquema populista no solo es top-down, o solo verticalista, según la cual esas demandas, frustradas, casi ciegas, solo las puede articular un liderazgo desde arriba. El liderazgo muchas veces es la expresión de esa articulación previa, horizontal. Ahora ya meto de lleno en el tema: el liderazgo, para seguir representando una voluntad creciente e incipiente no representada, para seguir trasladando y siendo el mensajero de la voluntad de A en un lugar donde A no está, tiene que escuchar y tiene que modificarse. Nosotros en la campaña electoral de Podemos escuchamos mucho. Los lemas, y los discursos, fueron mutando en relación con la escucha de lo que nos decía la gente en las redes, en los mítines, lo que nos pedían, las palabras que nos pedían más. Los sectores representados no 
son sectores privados de iniciativa política, de modo que en condiciones de libertad pueden decidir dejar de estar representados. Pueden declararse no representados de nuevo, abandonarse, hacer otra cosa, postular representaciones diferentes. No pierden nunca del todo la iniciativa política.

\section{AJ:Si eso fuera así, ¿por qué hay esta crisis de representación en España? ¿Por qué los que mandan ya no convencen?}

IE: Hay una visión, creo, muy eurocéntrica, según la cual a los pueblos menos maduros les pega tener líderes. Es normal. Si uno coge la cultura política que pueda imaginar como más horizontalista y más enemiga de los liderazgos, por ejemplo el anarquismo, la más fuerte dentro del movimiento obrero español, que explique qué era si no un líder Buenaventura Durruti, al que cuando muere le van a despedir cientos de miles de obreros a su entierro en Barcelona.

En torno al liderazgo, a veces es verdad que uno puede diagnosticar que a mayor existencia de instituciones, a mayores cauces organizativos, menor necesidad de los liderazgos de tipo carismático. Pero donde ha habido confianzas muy rotas, a veces el liderazgo sirve para construir desde arriba, en torno a una figura que le da un nombre nuevo a quienes hasta ayer no habían compartido nada. La gente que se identifica con Podemos, ayer no tenía ninguna etiqueta que les definiera. La última encuesta de CelesteTel para ElDiario.es dice que un $24,7 \%$ de los que declaran que votarían a Podemos provienen del Partido Popular. Esa gente no compartía ninguna etiqueta, entonces intentar reconducirla a una etiqueta anterior supone no entender lo que está sucediendo. Si no compartía ninguna etiqueta, ¿qué necesita? Nombres nuevos para identificarse.

Después está la construcción popular, la reivindicación de la soberanía popular y la posibilidad, luego, de construir institucionalidad nueva. Una vez construido el sujeto pueblo, que irrumpe en el Estado, diciendo que muchas cosas tienen que ser cambiadas. Todo tiene que sedimentar luego en nuevas formas de plasmar esa correlación de fuerzas, en esa pugna a veces amistosa, entre democracia y liberalismo. Que es una cosa que en Europa no se ha atendido mucho, y que en América Latina tienen mucho más teorizada. Quizá porque allí la tradición democrática y la tradición liberal no vinieron juntas, se pegaron a veces, y aquí parece que los dos términos sean la misma cosa. La tradición del liberalismo se ha ido comiendo paulatinamente la tradición democrática, al punto que los derechos individuales, el derecho de participar políticamente votando, y el derecho de que haya medios de comunicación, parece que ya definen la democracia. Yo diría que esto es condición necesaria para que haya democracia, pero también hay otras, por ejemplo, como el derecho a un sustento de ciudadano para que no 
tenga que vivir con miedo o tener que venderse, la posibilidad de ejercer la soberanía popular. Creo que ahí ha habido una pugna que tenemos poco teorizada, que ha permitido que los sectores más conservadores hayan ido produciendo un estrechamiento de lo democrático en nuestro sistema político.

\section{AJ: En la dirección de Podemos se concentra un potente capital intelectual, que en el día} a día va desarrollando la fusión de trabajo político, analítico y teórico, con una innovadora gestión mediática y comunicacional de curso que pinta vertiginoso. ¿Cómo se lleva todo esto, al mismo tiempo, con tan pocos recursos?

IE: Con ansiedad y con tristeza, porque reconocemos que para hacer política virtuosa en tiempos revueltos, para amar en tiempos revueltos, hace falta hacer, hace falta estudiar y hace falta pensar, hace falta atreverse y levantar la cabeza en la coyuntura. Pero el ciclo político acelerado en el que estamos, y el lugar central logrado por Podemos en la disputa política cotidiana, hace muy difícil leer, es muy difícil estudiar, porque además los ritmos de la reflexión del planteamiento político y de la intervención, los ritmos de la pelea en los medios, de la disputa día a día de la construcción interna son a veces diferentes. Y nosotros ahora, recientemente hemos tomado una decisión, de dar un paso atrás para dar dos pasos adelante, diría. Una parte de quienes estábamos teniendo portavocías de primer orden, no las dejamos de tener, pero nos descargamos un poco de esa tarea.

La experiencia Podemos es deudora de muchas cosas, del cambio cultural que se produce con el $15 \mathrm{M}$, de la lectura que algunos en particular hacemos de los procesos latinoamericanos y de la forma de pensar la política que ello nos da, de una política comunicativa que hacen otros compañeros que abre un espacio sin el cual todo esto no habría pasado de ser reflexiones teóricas. Es deudora de muchas cosas, entre ellas un grupo de investigadores, estudiantes y profesores, muchos de ellos en la Facultad de Ciencias Políticas, que empiezan a discutir. Sin tener en absoluto una hoja trazada, porque nadie tenía en la cabeza Podemos, pero donde sí empezaron a fraguarse herramientas de análisis con gente de distintos ambientes. Hay compañeros que se dedican a la cuestión de los medios de comunicación, otros se dedican a replantearnos la Transición y su relación con el sistema actual, otros piensan sobre las experiencias de cambio latinoamericanas, otros los movimientos sociales.

Todo eso hace una especie de puchero intelectual que va hirviendo a fuego lento y que fabrica un semillero de ideas muy rico. Del que luego hemos tirado a mucha velocidad. Pero lo 
que pasa es que esas ideas se consumen más rápido de lo que se producen, todo ese puchero que ha tardado cuatro o cinco años en producirse nos lo hemos comido en seis meses. Ahora hay que volver a hacerlo. Por gentes que todavía no conocemos, o que no estaban en eso, pero cuya aportación al trabajo y desarrollo intelectual vamos a necesitar, porque nunca debemos perder esa parte. $\mathrm{Y}$ en parte porque es necesario, si queremos ser capaces de construir un sujeto con la mayoría que está machacada, para una nueva mayoría que recupere las instituciones, la democracia, y ponga la política al servicio de la gente.

Si queremos ganar, hay que seguir pensando con la osadía de poder levantar la cabeza por encima de la coyuntura, de poder imaginarse cosas que quizá están ahí como posibilidad, como potencia. Hay que leerlo y hay que atreverse y no siempre es compatible con la coyuntura de la vida política, que tiende a encerrarte en circuitos muy pequeños. Cuando te alejas del movimiento magmático de las dinámicas sociales, ya no puedes intervenir en un sentido de transformación radical.

\section{AJ: Bueno te dejo trabajar Íñigo, muchas gracias por tu pedagogía política apasionada.}

IE: No, no, a gusto, interesante. Esto ayuda a pensar. 


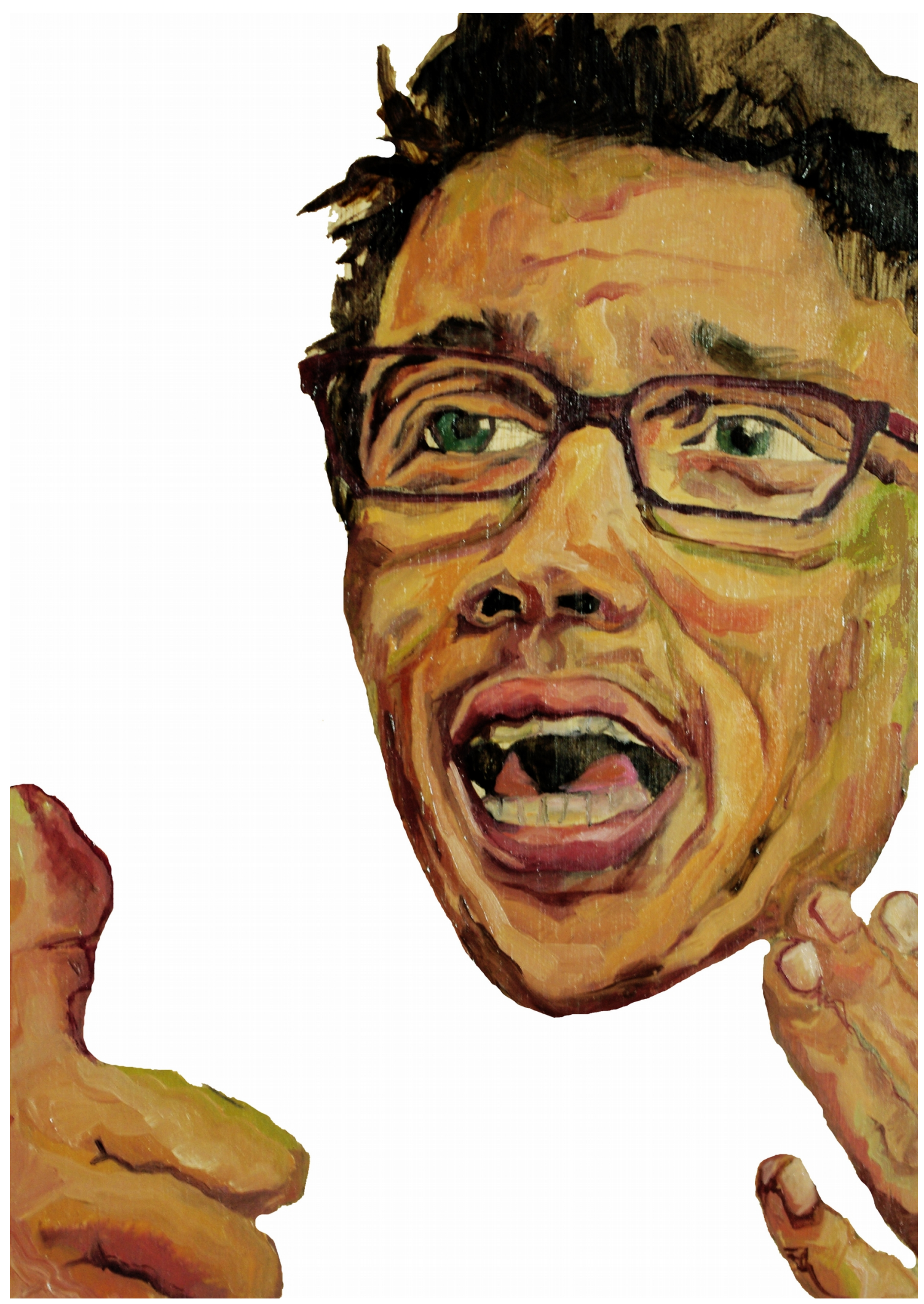

